\title{
Ultrasound Guidance for Interventional Pain Management of Lumbar Facet Joint Pain: An Anatomical and Clinical Study
}

\author{
Masoud Hashemi, ${ }^{1}$ Seyed Mohammad Jazayeri, ${ }^{2}$ Asadollah Saadat Niaki, ${ }^{3}$ Mahshid Nikooseresht, ${ }^{4,}$ \\ Alireza Hosseinpanah, ${ }^{5}$ Seyed Sajad Razavi, ${ }^{1}$ Farshad Farivar, ${ }^{1}$ and Farideh Shahandeh ${ }^{6}$ \\ ${ }^{1}$ Department of Anesthesiology, Akhtar Hospital, Shahid Beheshti University of Medical Sciences, Tehran, Iran \\ ${ }^{2}$ Department of Orthopedics, Akhtar Hospital, Shahid Beheshti University of Medical Sciences, Tehran, Iran \\ ${ }^{3}$ Department of Anesthesiology, Imam Hossein Hospital, Shahid Beheshti University of Medical Sciences, Tehran, Iran \\ ${ }^{4}$ Department of Anesthesiology, Beasat Hospital, Hamedan University of Medical Sciences, Hamedan, Iran \\ ${ }^{5}$ Fellowship of Pain, Tehran, Iran \\ ${ }^{6}$ Department of Radiology, Akhtar Hospital, Shahid Beheshti University of Medical Sciences, Tehran, Iran \\ "Corresponding author: Mahshid Nikooseresht, Department of Anesthesiology, Beasat Hospital, Hamedan University of Medical Sciences, Hamedan, Iran. Tel/Fax: \\ +98-8112651515, E-mail: nikoo_mahshid@yahoo.com
}

Received 2015 November 18; Revised 2015 December 07; Accepted 2016 January 18.

\begin{abstract}
Background: There is a growing trend in ultrasound use in interventional pain management. Recently, the ease of use and clinical benefits of lumbar medial branch nerve block under ultrasound guidance have been identified.

Objectives: In this study, we assessed the relevant anatomy and sonoanatomy of these specific interventional techniques. We also evaluated the feasibility and success rates of ultrasound guided lumbar medial branch nerve block.

Patients and Methods: Thirty patients with facet joint pain who were referred to the Akhtar hospital pain clinics between 2011 and 2012 were evaluated. Eighty-nine ultrasound-guided lumbar medial branch nerve blocks were performed. The target point for the lumbar nerve block was the cephalad margin of the transverse process groove in the vicinity of the superior articular process. Carm fluoroscopy was performed to confirm the needle location. Pain levels were measured by a visual analog scale ( 0 - 10 scale), the Oswestry disability index ( 0 - 5 scale), and patient satisfaction scores ( 0 - 3 scale). The patients were followed for 6 weeks.

Results: The success rate was $98 \%$ (87/89 blocks), which was due to our use of ultrasound guided needle placement for the correct positioning of the needles. The mean procedural time was $5.9 \pm 1$ minutes. The average time of needle insertion was $4 \pm 1$ minutes. The pain intensity significantly improved from an initial value of 5 to 2.8 in the final follow-up $(\mathrm{P}=0.0001)$. The oswestry disability index score significantly improved from 33.9 to 18.3 in the final follow-up $(\mathrm{P}=0.0001)$. Patient satisfaction significantly improved from poor satisfaction immediately after the medial branch nerve block to excellent satisfaction in the final follow-up $(P=0$. 0001). Analgesic requirements were also significantly reduced after 6 weeks of follow-up $(P=0.046)$.

Conclusion: Lumbar medial branch nerve block under ultrasound guidance was associated with high rates of treatment success and excellent treatment outcomes for facet joint pain. It is also feasible and administers no radiation. Thus, ultrasound-guided procedures can be used instead of conventional methods.
\end{abstract}

Keywords: Ultrasound, Lumbar Facet Joint Pain, Medial Branch Block

\section{Background}

Lumbar facet joints are one of the major contributors of chronic low-back pain, which can potentially lead to referred pain (1). Lumbar facet joint pain cannot be diagnosed by either physical assessments (2) or X-rays (3). A lumbar medial branch block is a diagnostic block for a lumbar facet joint pain diagnosis (4-7).

Years ago, physicians palpated anatomic landmarks for the insertion of the guide needle. More recently, fluoroscopic or computed tomography has been routinely used for guidance in our institution. Currently, slightly invasive and imaging-guided techniques have joined the tools used in pain management. The reason for their increased use is mainly due to their ease of use and high success rates in controlling chronic pain, which is of interest to practitioners $(8,9)$.

The use of ultrasound in pain medicine is rapidly growing, especially in various types of procedures for interventional pain management. Much of the recent research has investigated the procedures used under ultrasound guidance (10).

Fluoroscopy showing bone structure is a common practice, but cannot show the adjacent building. Ultrasound-guided techniques that show the communications between anatomical structures improve the accuracy of the procedure.

Lately, facet nerve blocks under ultrasound guidance 
have been done for chronic pain management. Because of the cost and lack of radiation exposure, the use of ultrasound guidance has been considered more beneficial (11, 12). The use of ultrasound in interventional pain management can also offer more advantages than that of conventional fluoroscopy $(13,14)$. Furthermore, ultrasound guidance can be used in various types of nerve blocks.

\section{Objectives}

The purposes of this study were to examine the relationship between bone anatomy and sonoanatomy using ultrasound guidance, and to evaluate the feasibility and success rates of ultrasound-guided lumbar medial branch nerve blocks.

\section{Patients and Methods}

This study was a prospective clinical trial (IRCT ID: IRCT201306168435N6). The study protocol was approved by the Shahid Beheshti University of Medical Science ethics committee. We evaluated 60 patients with chronic low back pain in the facet joint region who were referred to the pain clinics of Akhtar hospitals between 2011 and 2012. The patients with the following features were included: age $>$ 18 years, symptoms of chronic low back pain ( $>3$ months), persistent back pain with or without pain radiating to the leg, focal lumbar tenderness and hyperextension pain, the absence of neural tube defects, contraindicated for lumbar surgery, an absence of radicular syndrome, and a failure to respond to traditional treatments. Patients were excluded if they had a malignancy, a coagulation disorder, or an allergy to the local anesthetic solutions. Furthermore, patients with behavioral problems, pregnancy, localized skin infection at the surgical site, spinal deformities, or patients who were uncooperative due to psychiatric disorders were also excluded.

All blocks were performed by one pain specialist. Patients in the prone position with lordosis support is destined to compensate. With a 7.5- to $12-\mathrm{MHz}, 30-\mathrm{mm}$, broadband curved array transducer (HS-2600, Honda electronics Inc., Honda, Japan) targeted the lumbar vertebral joint with the longitudinal axis of the sacrum as measured by ultrasound was a turning point (15).

After identifying the target beads, to see the transverse and superior articular processes (SAP) and to obtain a view of the cross shaft became ultrasound probe to 90 degrees to show the distance from the cephalad margin of the adjacent groove side to the SAP(16), and the nearest skin surface was measured using an ultrasound caliper. Local anesthesia was performed with a $1 \%$ lidocaine injection ( $1 \mathrm{~mL})$. A 21-gauge spinal needle with a length of $9 \mathrm{~cm}$ to the ultrasound disk and a 45- to 60-degree angle to the lateral and medial skin to its tip was inserted into the target bone is felt, driven. A brokerage ultrasound needle appropriate for the target site points out C-arm was performed to evaluate the correct insertion of the needle. The correct needle insertion at the target site was in accordance with the international guidelines of the spinal society forum. The correct needle insertion in the anterior posterior view was at the posterior edge of the SAP and above the Scotty dog's eye in the ultrasound diagonal view (15). If in view of the ultrasound needle was not put in the correct place, under the $C$ arm to guide the insertion needle has been modified, then the injection was carried out (Figures 1 and 2).

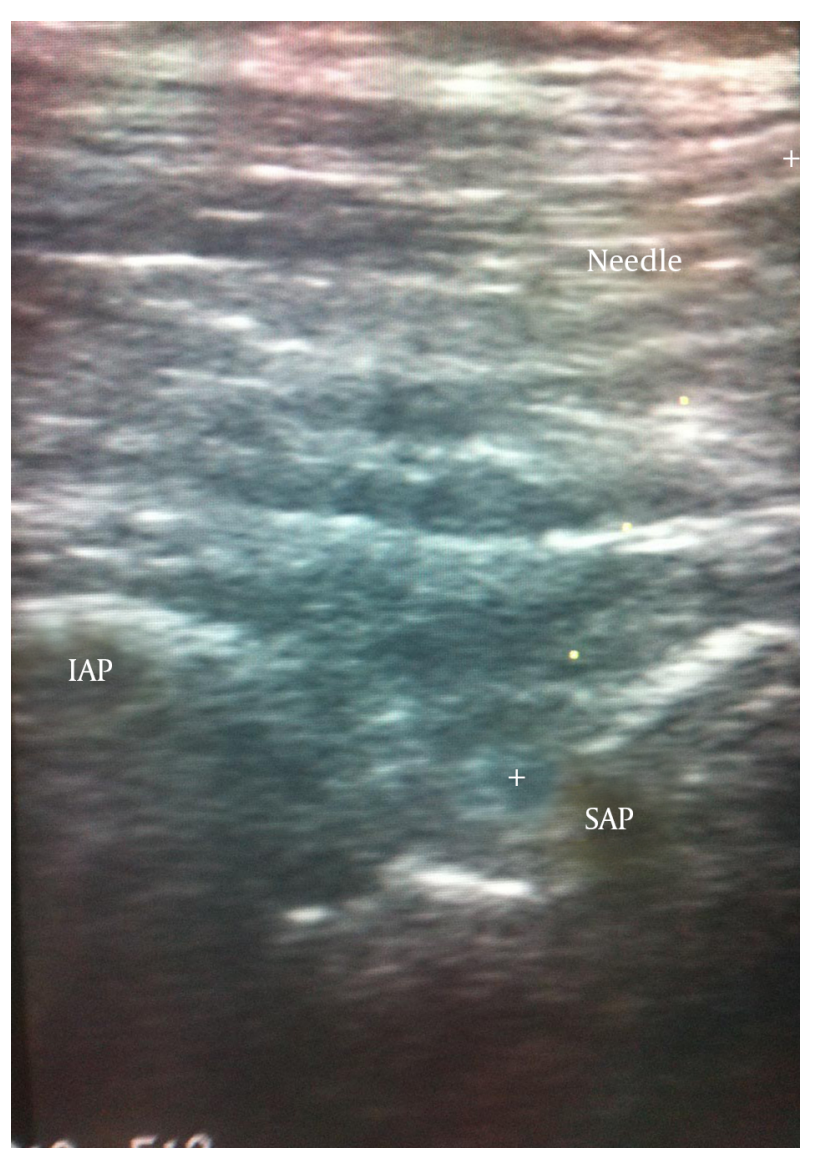

Figure 1. IAP, inferior articular process; SAP, superior articular process

The combination of triamcinolone acetonide (40 mg) and $1 \%$ lidocaine $(1 \mathrm{~mL})$ at the L1-S1 levels on the right/left sides was injected for the treatment of lumbar facet joint pain.

We recorded demographic characteristics including sex, age, body weight, height, and body mass index (BMI). 


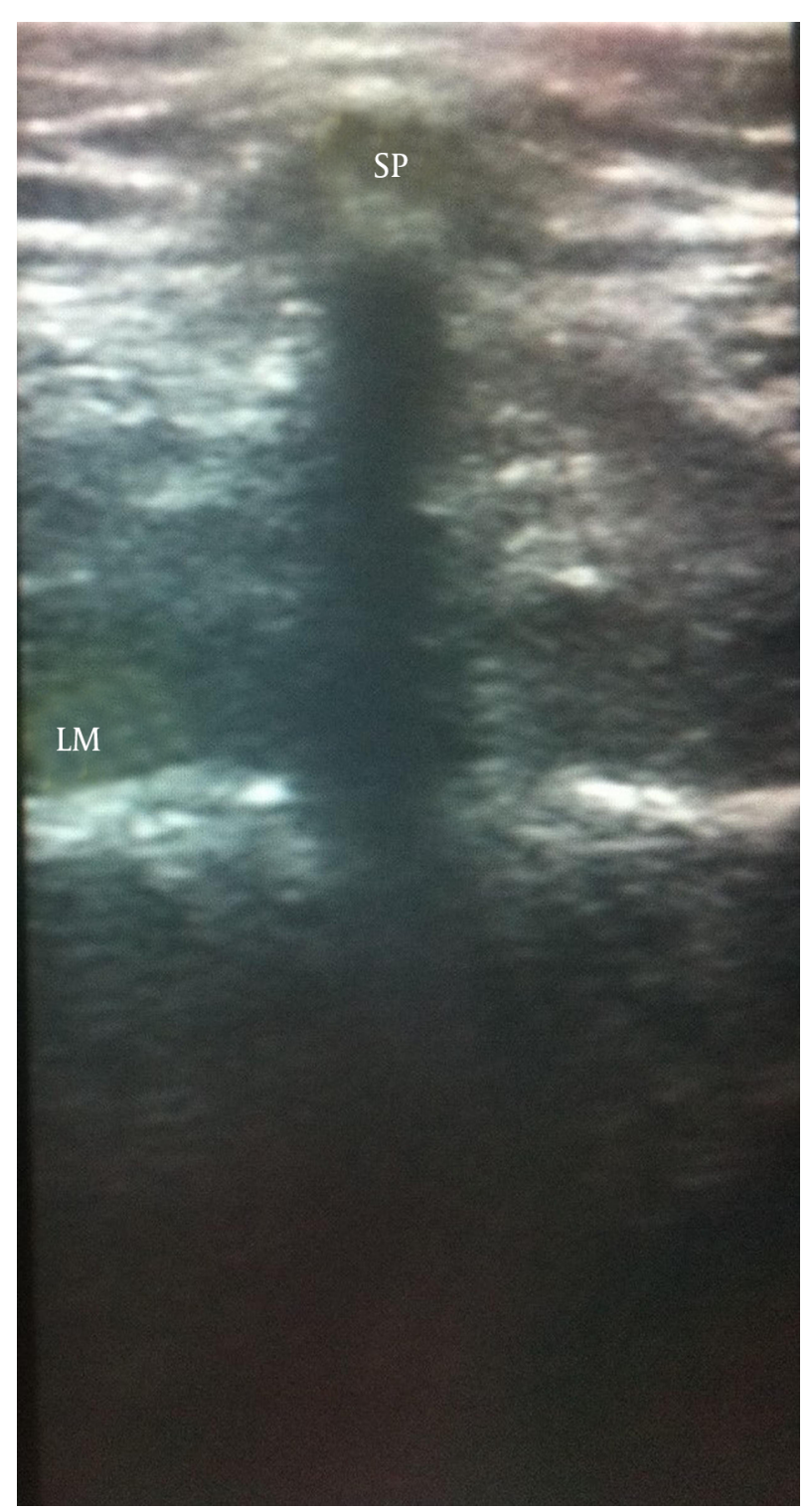

Figure 2. LM, Lamina; SP, Spinous process

Low back pain was assessed before, immediately after, and at 1 week, 4 weeks, and 6 weeks after the block by a visual analog scale (VAS; $0=$ no pain; $10=$ worst pain). The oswestry disability index (ODI) score was assessed before, immediately after, and at 1 week, 4 weeks, and 6 weeks after the block using a 5 -point scale ( $0=$ no disability; $5=$ worst disability) (17). Patient satisfaction of pain control was assessed immediately after, and at 1 week, 4 weeks, and 6 weeks after the block using a 4-point scale $(0=$ poor satisfaction; 3 = excellent satisfaction). If the patient reported a VAS $>4$ for pain, he/she received a anti-inflammatory drug
(NSAID)

The procedure time from the beginning brokerage needle to the end of the injection was determined and recorded. The needle insertion, defined as the time from its insertion into the skin, was confirmed by fluoroscopic methods. If the needle tip was off-target and not visible by ultrasound, the attempt was considered a failure.

The sample size for this study had an $80 \%$ power. Data was evaluated with the statistical package for the social sciences (SPSS) software version 19 (SPSS Inc., Chicago, IL, USA). Normal distribution of samples was determined using Kolmogorov-Smirnov test. Descriptive parameters included frequency, percentage, mean, and standard deviation (SD). Comparisons of qualitative variables or quantitative variables without normal distribution were performed by the Wilcoxon and Friedman's tests, while repeated measures analysis of variance (ANOVA) were performed for comparison of quantitative variables. A P value of $<0.05$ was considered statistically significant.

\section{Results}

Thirty patients received ultrasound-guided medial branch blocks for lumbar facet joint pain. The mean age of the patients was $36.2 \pm 10.8$ (range: 18 - 58) years. Sixteen patients were men (53.3\%) and 14 (46.7\%) were women. The mean BMI was $24.7 \pm 2.6 \mathrm{~kg} / \mathrm{m}^{2}$. Ultrasound-guided medial branch blocks were performed in 11 patients (36.7\%) for $\mathrm{L} 3 / 4$ and $\mathrm{L} 4 / 5$; 11 patients (36.7\%) for $\mathrm{L} 4 / 5$ and $\mathrm{L} 5 / \mathrm{S} 1 ; 5$ patients (16.7\%) for $\mathrm{L} 2 / 3$ and $\mathrm{L} 3 / 4$; and 3 patients (10\%) for $\mathrm{L} 1 / 2$ and $\mathrm{L} 2 / 3$. In terms of the practicality of cross-axis ultrasound to guide the needle in the 30 patients, all 89 medial branch blocks were performed. All needles were directed to the correct place, and the medial branches blocked included 30 for L3, 31 for L4, and 28 for L5. In 15 blocks, the needles were not confirmed in the longitudinal paravertebral view, then up and down along the groove in the crossaxis displays walked. Among the 89 blocks, 87 needles were placed in the correct position, resulting in a success rate of $98 \%$. Only two needles needed minor adjustments after confirmation by the C-arm; these needles were slightly shifted laterally on the posterior side of the transverse process. The mean procedural time was $5.9 \pm 1$ minutes. The time required to insert the needle was $4 \pm 1$ minutes. There were no procedure-related complications. The mean VAS score was significantly reduced from 5 points (range: 4 - 8 points) preoperatively to 2.8 points (range: 1 - 4 points) at the final follow-up examination $(\mathrm{P}=0.0001)$ (Figure 3$)$.

The mean ODI was significantly reduced from 33.9 (range: 22 - 48) preoperatively to 18.3 (range: 10 - 26) at the final follow-up examination $(\mathrm{P}=0.0001)$ (Figure 4). 


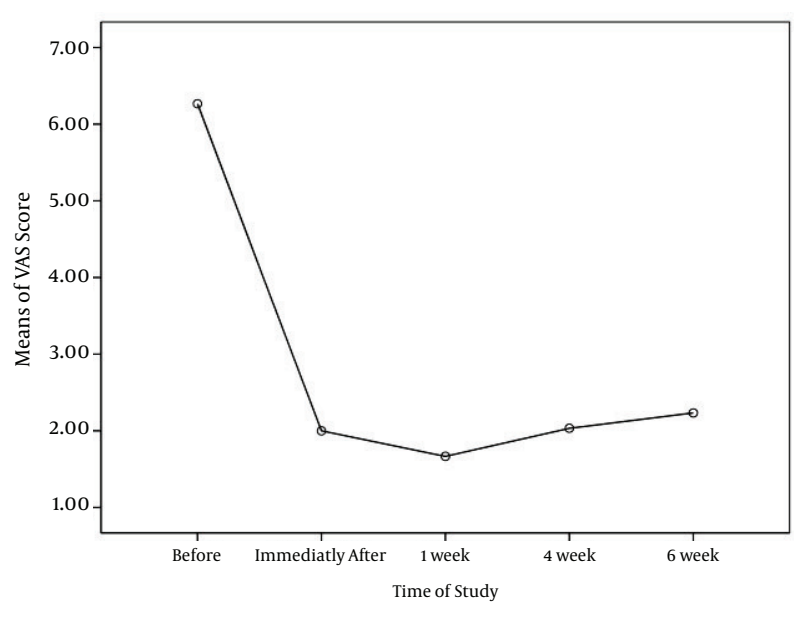

Figure 3. The visual analog scale (VAS) scores changed at various times throughout the study $(\mathrm{P}=0.0001)$

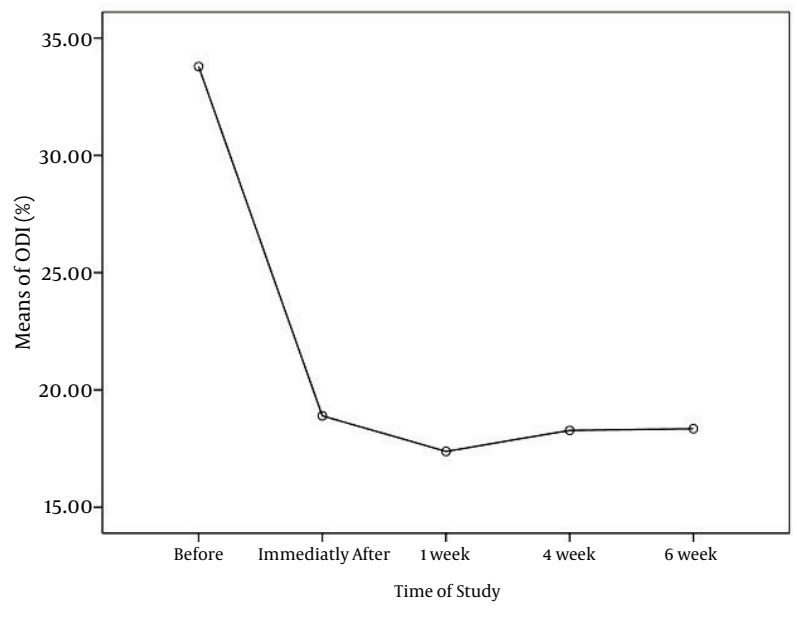

Figure 4. The oswestry disability index (ODI) scores changed at various times throughout the study $(\mathrm{P}=0.0001)$

Patient satisfaction was significantly improved from poor satisfaction immediately after the medial branch block to excellent satisfaction at the final follow-up examination $(\mathrm{P}=0.0001)$.

Furthermore, analgesic requirements were significantly reduced at the final follow-up examination ( $\mathrm{P}=$ 0.046).

\section{Discussion}

Lumbar facet joint pain cannot be diagnosed based on physical or radiology results alone $(2,3)$. A standard diagnostic block for facet joint pain is a medial branch block on the dorsal ramus of a spinal nerve that is adjacent to a facet joint, which is necessary to confirm the site of the pain (14).

To improve the precision of needle placements and reduce complication rates, fluoroscopic or computed tomography guidance has been considered compulsory (18). However, these techniques are associated with radiation exposure and higher costs (18). Alternatively, ultrasound guidance can be used for needle placement. Ultrasonography plays an important role in aiding regional nerve and peripheral-nerve blocks (19-22). Greher et al. reported a high success rate, clinical feasibility, and usefulness of ultrasound-guided lumbar medial branch blocks $(11,23)$. High-quality sonographic views depend on the technical expertise of the specialty pain physician and a specialized knowledge of radiology. The block used in this study was easy and it was visible in the joint. The needle's cephalocaudal angle must be forward while the needle is in the groove, and the needle under the ultrasound waves is placed at an angle that is visible on the facade of the transverse axis. One problem of the cross-axis view concerns the direction of the needle at the target site since this view does not give the necessary information to the doctor. To increase the success rate of medial branch blocks, the needle tip is placed in the groove margin cephalad. In our experience, to obtain a cross profile interspinous space probe axis and move up and down where target site first seen and the ease of insertion needle in the margin of cephalad. In our experience, for the ease of insertion needle in the margin of cephalad, we move up and down probe axis where target site first seen. However, in view of the longitudinal axis of the needle tip approval often need to spend. Therefore, if the quality is poor, we set walk off the needle track in order to make it to the cephalad intervertebral foramen weaken and then to caudal in the walked bone. This movement in the cross axis view needle groove must be visible. In our study, we used a corticosteroid for the facet joint injections. Ultrasound can be used for corticosteroid injections for diagnostic blocks. The literature on the use of steroids in facet joint injections is controversial. Early trials failed to show improvements using facet joint injections with steroids. However, recent studies identified good outcome treatments with steroid injections $(24,25)$. Despite the suspicion surrounding steroid actions, some studies have subscribed to this treatment for clinical pain symptoms in their practices $(26,27)$.

We applied an injection volume of $1 \mathrm{~mL}$. The optimal volume for a facet joint injection is still controversial, however, the usual amount for facet joints is $1-2 \mathrm{~mL}$. In our study, during the short term follow up, pain relief was observed after facet joint injections, a finding confirmed by other studies (28). The results of facet joint injections can probably be improved with ultrasound guidance on cer- 
tain patients to provide a better response to interventions. Most pain practitioners are largely used for facet joint injections. Also, when injections are performed under imaging guidance, complication rates are decreased and better outcomes are obtained $(24,29)$. Thus, the use of preciseness and safety equipment, such as ultrasound without radiation, have been highly requested. Ultrasound can produce improvements in clinical symptoms. In our study, patient satisfaction as well as good outcomes with significant differences in the degree of pain control and functional status improvement were obtained. Ultrasound is widely available, and in many patients, it is associated with a high degree of accuracy.

In conclusion, lumbar medial branch nerve blocks under ultrasound guidance can be used with a high rate of treatment success. Ultrasound is an invaluable tool for imaging the structure of soft tissues and bones, to guide needles, and to verify the progress of substances injected around the target. Moreover, it is a radiation-free method and ultrasound guidance can be used to replace traditional methods.

\section{Acknowledgments}

We would like to thank our pain ward colleagues who helped our research in many ways.

\section{Footnotes}

Authors' Contribution: Masoud Hashemi developed the original idea and the protocol, abstracted and analyzed data, wrote the manuscript, and is the guarantor. Mahshid Nikuseresht and other authors contributed to the development of the protocol, the abstracted data, and prepared the manuscript.

Financial Disclosure: None.

Funding/Support: This study was not supported by a grant from the Akhtar hospital or the Shahid Beheshti University of Medical Sciences.

\section{References}

1. Schwarzer AC, Wang SC, Bogduk N, McNaught PI, Laurent R. Prevalence and clinical features of lumbar zygapophysial joint pain: a study in an Australian population with chronic low back pain. Ann Rheum Dis. 1995;54(2):100-6. [PubMed: 7702395].

2. Schwarzer AC, Aprill CN, Derby R, Fortin J, Kine G, Bogduk N. Clinical features of patients with pain stemming from the lumbar zygapophysial joints. Is the lumbar facet syndrome a clinical entity?. Spine (Phila Pa 1976). 1994;19(10):1132-7. [PubMed: 8059268].

3. Schwarzer AC, Wang SC, O'Driscoll D, Harrington T, Bogduk N, Laurent $\mathrm{R}$. The ability of computed tomography to identify a painful zygapophysial joint in patients with chronic low back pain. Spine (Phila Pa 1976). 1995;20(8):907-12. [PubMed: 7644955].
4. Boswell MV, Colson JD, Sehgal N, Dunbar EE, Epter R. A systematic review of therapeutic facet joint interventions in chronic spinal pain. Pain Physician. 2007;10(1):229-53. [PubMed:17256032].

5. Cohen SP, Raja SN. Pathogenesis, diagnosis, and treatment of lumbar zygapophysial (facet) joint pain. Anesthesiology. 2007;106(3):591-614. [PubMed: 17325518].

6. Sowa G. Facet-mediated pain. Dis Mon. 2005;51(1):18-33. doi: 10.1016/j.disamonth.2004.12.005. [PubMed:15805977].

7. Yin W, Willard F, Carreiro J, Dreyfuss P. Sensory stimulation-guided sacroiliac joint radiofrequency neurotomy: technique based on neuroanatomy of the dorsal sacral plexus. Spine (Phila Pa 1976). 2003;28(20):2419-25. doi: 10.1097/01.BRS.0000085360.03758.C3. [PubMed: 14560094].

8. Carrino JA, Morrison WB, Parker L, Schweitzer ME, Levin DC, Sunshine JH. Spinal injection procedures: volume, provider distribution, and reimbursement in the U.S. medicare population from 1993 to 1999. Radiology. 2002;225(3):723-9. doi:10.1148/radiol.2253011401. [PubMed: 12461252].

9. Kim PS. Role of injection therapy: review of indications for trigger point injections, regional blocks, facet joint injections, and intraarticular injections. Curr Opin Rheumatol. 2002;14(1):52-7. [PubMed: 11790997].

10. Peng PW, Narouze S. Ultrasound-guided interventional procedures in pain medicine: a review of anatomy, sonoanatomy, and procedures: part I: nonaxial structures. Reg Anesth Pain Med. 2009;34(5):458-74. doi: 10.1097/AAP.0b013e3181aea16f. [PubMed: 19920421].

11. Greher M, Scharbert G, Kamolz LP, Beck H, Gustorff B, Kirchmair L, et al. Ultrasound-guided lumbar facet nerve block: a sonoanatomic study of a new methodologic approach. Anesthesiology. 2004;100(5):1242-8. [PubMed: 15114223].

12. Shim JK, Moon JC, Yoon KB, Kim WO, Yoon DM. Ultrasoundguided lumbar medial-branch block: a clinical study with fluoroscopy control. Reg Anesth Pain Med. 2006;31(5):451-4. doi: 10.1016/j.rapm.2006.06.246. [PubMed:16952818].

13. Wallace MA, Fukui MB, Williams RL, Ku A, Baghai P. Complications of cervical selective nerve root blocks performed with fluoroscopic guidance. AJR Am J Roentgenol. 2007;188(5):1218-21. doi: 10.2214/AJR.04.1541. [PubMed:17449763].

14. Rozin L, Rozin R, Koehler SA, Shakir A, Ladham S, Barmada M, et al. Death during transforaminal epidural steroid nerve root block (C7) due to perforation of the left vertebral artery. Am J Forensic Med Pathol. 2003;24(4):351-5. doi:10.1097/01.paf.0000097790.45455.45. [PubMed: 14634474].

15. Bogduk N. International Spinal Injection Society guidelines for the performance of spinal injection procedures. Part 1: Zygapophysial joint blocks. Clin J Pain. 1997;13(4):285-302. [PubMed: 9430809].

16. Dreyfuss P, Schwarzer AC, Lau P, Bogduk N. Specificity of lumbar medial branch and L5 dorsal ramus blocks. A computed tomography study. Spine (Phila Pa 1976). 1997;22(8):895-902. [PubMed: 9127924].

17. Intensity P. Modified oswestry low back pain disability questionnaire. Physiotherapy. 1980;66:271-3.

18. Zhou Y, Singh N, Abdi S, Wu J, Crawford J, Furgang FA. Fluoroscopy radiation safety for spine interventional pain procedures in university teaching hospitals. Pain Physician. 2005;8(1):49-53. [PubMed: 16850042].

19. Peterson MK, Millar FA, Sheppard DG. Ultrasound-guided nerve blocks. BrJAnaesth. 2002;88(5):621-4. [PubMed: 12066995].

20. Marhofer P, Greher M, Kapral S. Ultrasound guidance in regional anaesthesia. Br J Anaesth. 2005;94(1):7-17. doi: 10.1093/bja/aei002. [PubMed: 15277302].

21. Kirchmair L, Entner T, Kapral S, Mitterschiffthaler G. Ultrasound guidance for the psoas compartment block: an imaging study. Anesth Analg. 2002;94(3):706-10. [PubMed: 11867402] table of contents. 
22. Kirchmair L, Entner T, Wissel J, Moriggl B, Kapral S, Mitterschiffthaler G. A study of the paravertebral anatomy for ultrasound-guided posterior lumbar plexus block. Anesth Analg. 2001;93(2):477-81. [PubMed: 11473883] 4th contents page.

23. Greher M, Kirchmair L, Enna B, Kovacs P, Gustorff B, Kapral S, et al. Ultrasound-guided lumbar facet nerve block: accuracy of a new technique confirmed by computed tomography. Anesthesiology. 2004;101(5):1195-200. [PubMed: 15505456].

24. Aguirre DA, Bermudez S, Diaz OM. Spinal CT-guided interventional procedures for management of chronic back pain. J Vasc Interv Radiol. 2005;16(5):689-97. doi: 10.1097/01.RVI.0000156193.94573.48. [PubMed: 15872324].

25. Pneumaticos SG, Chatziioannou SN, Hipp JA, Moore WH, Esses SI. Low back pain: prediction of short-term outcome of facet joint injection with bone scintigraphy. Radiology. 2006;238(2):693-8. doi: 10.1148/radiol.2382041930. [PubMed: 16436824].

26. Lippitt AB. The facet joint and its role in spine pain. Management with facet joint injections. Spine (Phila Pa 1976). 1984;9(7):746-50. [PubMed: 6239395].

27. Lewinnek GE, Warfield CA. Facet joint degeneration as a cause of low back pain. Clin Orthop Relat Res. 1986(213):216-22. [PubMed: 2946505]

28. Gorbach C, Schmid MR, Elfering A, Hodler J, Boos N. Therapeutic ef ficacy of facet joint blocks. AJR Am J Roentgenol. 2006;186(5):1228-33. doi: 10.2214/AJR.04.1042. [PubMed: 16632710].

29. Silbergleit R, Brunberg JA, Patel SC, Mehta BA, Aravapalli SR. Imaging of spinal intradural arachnoid cysts: MRI, myelography and CT. Neuroradiology. 1998;40(10):664-8. [PubMed: 9833898]. 